\title{
INDEX AND TOTAL CURVATURE OF SURFACES WITH CONSTANT MEAN CURVATURE
}

\author{
MANFREDO P. DO CARMO AND ALEXANDRE M. DA SILVEIRA
}

(Communicated by Jonathan M. Rosenberg)

\begin{abstract}
We prove an analogue, for surfaces with constant mean curvature in hyperbolic space, of a theorem of Fischer-Colbrie and Gulliver about minimal surfaces in Euclidean space. That is, for a complete surface $M^{2}$ in hyperbolic 3-space with constant mean curvature 1, the (Morse) index of the operator $L=\Delta-2 K$ is finite if and only if the total Gaussian curvature is finite.
\end{abstract}

\section{INTRODUCTION}

Let $x: M^{2} \rightarrow R^{3}$ be a minimal immersion into the Euclidean space $R^{3}$, and let $K, d A$, and $\Delta$ respectively be the Gaussian curvature, the area element, and the Laplacian, of the induced metric. Recently, the following result that relates the total curvature $\int_{M}(-K) d A$ of $M$ with the (Morse) index $\operatorname{Ind}_{M} L$ for the operator $L=\Delta-2 K$ was proved.

Theorem A (D. Fischer-Colbrie [2], R. Gulliver [3]). Let $x: M^{2} \rightarrow R^{3}$ be a complete minimal surface. Then

$$
\text { Ind }_{M} L \text { is finite } \Leftrightarrow \int_{M}(-K) d A \text { is finite. }
$$

Here $\operatorname{Ind}_{M} L$ is defined as follows. Let $D \subset M$ be a domain with compact closure $\bar{D}$ and let $\mathscr{F}(D)$ be the set of piecewise smooth functions in $M$ whose supports are contained in $\bar{D}$. An eigenfunction $g \in \mathscr{F}(D)$ of $L$ satisfies the equation $L g+\lambda g=0$ for some (real) $\lambda$ that is called an eigenvalue of $L$; the multiplicity of $\lambda$ is the (finite) dimension of the vector space of functions that satisfy the above equation. The index $\operatorname{Ind}_{D} L$ is the number (counted with multiplicities) of negative eigenvalues of $L$ in $D$. By letting $M$ be exhausted by a sequence $D_{0} \subset D_{1} \subset \cdots \subset D_{t} \subset M$ of increasing domains, it can be shown that

$$
\sup _{t}\left(\operatorname{Ind}_{D_{t}} L\right)=\operatorname{Ind}_{M} L
$$

is independent of the choices made; it is called the index of $L$ in $M$.

Received by the editors November 17, 1988 and, in revised form, February 26, 1990.

1980 Mathematics Subject Classification (1985 Revision). Primary 53A10; Secondary 53C42. 
The geometric significance of the operator $L$ is that minus the quadratic form associated to it

$$
I(f)=-\int_{M}\left(f \Delta f-2 K f^{2}\right) d A
$$

is, modulo a constant factor, the second derivative of the area function of a normal variation given by the function $f$. Thus $\operatorname{Ind}_{D} L$ measures that "number of variations" in $D$ that decrease area.

We want to extend Theorem A to the following situation. Let $H^{3}(-1)$ be the three-dimensional hyperbolic space of constant sectional curvature -1 , and let $x: M^{2} \rightarrow H^{3}(-1)$ be a surface in $H^{3}(-1)$ with constant mean curvature $H=1$. Such surfaces were extensively studied in a recent paper by R. Bryant [1], and they share many properties with minimal surfaces in $R^{3}$. For instance, the induced metric $d s^{2}$ has Gaussian curvature $K \leq 0$, and $K$ vanishes only at isolated points of $M$. Further, away from points where $K=0$, the metric $d \tilde{s}^{2}=(-K) d s^{2}$ has curvature $\widetilde{K}=1$, and the second derivative of the area function for a normal variation given by $f \in \mathscr{F}(D)$ is

$$
I(f)=-\int_{M}\left(f \Delta f-2 K f^{2}\right) d A .
$$

Thus the operator $L=\Delta-2 K$ on $M$ has the same geometric meaning as before. The main result of this paper is that Theorem A holds in this situation.

Theorem B. Let $x: M^{2} \rightarrow H^{3}(-1)$ be a complete surface with constant mean curvature $H=1$. Then

$$
\operatorname{Ind}_{M} L \text { is finite } \Leftrightarrow \int_{M}(-K) d A \text { is finite. }
$$

\section{Preliminary CONSIDERATIONS}

An interesting remark about Theorem $A$ is that the implication $\Rightarrow$ is actually an intrinsic result. This was observed by Gulliver and Lawson [4] and is essentially contained in the proof of Theorem A given by Fischer-Colbrie in [2]. More specifically, we have the following result:

Theorem C (see, e.g., A. M. da Silveira [6]). Let $M^{2}$ be a surface with a complete Riemannian metric $d s^{2}$. Let $q \geq 0$ be a smooth function on $M$, and consider the operator $L=\Delta+q-K$, where $\Delta$ and $K$ are the Laplacian and the Gaussian curvature of $d s^{2}$. Assume that $\operatorname{Ind}_{M} L<\infty$. Then $M$ is conformally equivalent to a compact Riemann surface minus finitely many points, and $\int_{M} q d A<\infty$. B.

By setting $q=-K$, we obtain the implication $\Rightarrow$ for both Theorems A and

On the other hand, all the available proofs of the implication $\Leftarrow$ in Theorem A make essential use of the Gauss map of the immersion. Since $\int_{M}(-K) d A<$ $\infty$, it follows from a result of Huber [5] that $M$ is conformally equivalent to a 
compact Riemann surface $M_{c}$ punctured at finitely many points $p_{1}, \ldots, p_{k}$. The fact that the Gauss map extends across such punctures is then used.

In the present case, $x: M^{2} \rightarrow H^{3}(-1)$, there is a natural Gauss map to be considered that, when $x$ has constant mean curvature $H=1$, has similar properties to those of the Gauss map of a minimal surface in $R^{3}$ (cf. [1]). Since Huber's theorem is intrinsic, the condition that the total curvature is finite once more implies that $M$ is conformally equivalent to $M_{c}-\left\{p_{1}, \ldots, p_{k}\right\}$. However, as shown by various examples (cf. [1, Example 1]), such a Gauss map may no longer extend across the punctures.

The main point of the proof of Theorem $B$ is to show that the proof of the implication $\Leftarrow$ can be made independent of the Gauss map. The proof will also apply to complete minimal surfaces in $R^{3}$ (see Remark 2 below).

\section{Proof of Theorem B}

Since $\int_{M}(-K) d A<\infty, M$ is conformally equivalent to $M_{c}-\left\{p_{1}, \ldots, p_{k}\right\}$, where $M_{c}$ is a compact Riemann surface and $p_{i} \in M_{c}, i=1, \ldots, k$. We will assume that there is only one $p_{i}=p$; i.e., that there is a conformal diffeomorphism $F: M_{c}-\{p\} \rightarrow M$. The general case will be treated similarly.

Let $d s^{2}$ be the metric induced by $x$ on $M$, and let $d s_{c}^{2}$ be a metric on $M_{c}$ compatible with the conformal structure of $M_{c}$. Then $F^{*} d s^{2}=\lambda d s_{c}^{2}$, where $\lambda$ is a real positive function on $M_{c}-\{p\}$. By the completeness of $d s^{2}$, $\lim \lambda\left(p_{n}\right)=\infty$, for a sequence $\left\{p_{n}\right\} \rightarrow p, p_{n} \in M_{c}-\{p\}$.

Frequently we will use the notation loosely and denote by the same symbol $f$ both a function defined on $M$ and the composite $f \circ F$ defined on $M_{c}-\{p\}$.

Consider the operator $L_{c}=\Delta_{c}-2 K \lambda$ on $M_{c}-\{p\}$, where $\Delta_{c}$ is the Laplacian of $d s_{c}^{2}$ and $K$ is considered a function on $M_{c}-\{p\}$. Let $f$ be a function on $M$ with compact support, and denote by $d A_{c}=(1 / \lambda) F_{*} d A$ the area element of $d s_{c}^{2}$. Then

$$
\begin{aligned}
I_{L}(f) & =-\int_{M}\left(f \Delta f-2 K f^{2}\right) d A=\int_{M}\left(|\nabla f|^{2}+2 K f^{2}\right) d A \\
& =\int_{M_{c}}\left(\left|\nabla_{c} f\right|^{2}+2 K \lambda f^{2}\right) d A_{c}=I_{L_{c}}(f),
\end{aligned}
$$

where $\nabla f$ and $\nabla_{c} f$ are the gradients of $f$ in the metrics $d s^{2}$ and $d s_{c}^{2}$, respectively. Thus we must show that the index of $L_{c}$ in $M_{c}-\{p\}$ is finite. Notice that, since the total curvature is finite, $\lim K\left(p_{n}\right)=0$, for a sequence $\left\{p_{n}\right\} \rightarrow p, p_{n} \in M_{c}-\{p\}$.

Now let

$$
d \tilde{s}^{2}=(-K) d s^{2} .
$$

Then $d \tilde{s}^{2}$ is a pseudometric in $M$ (pseudometric here means that $d \tilde{s}^{2}$ can be zero only at isolated points) that has Gaussian curvature $\widetilde{K} \equiv 1$ and finite area. Therefore

$$
F^{*} d \tilde{s}^{2}=F^{*}\left((-K) d s^{2}\right)=-K \lambda d s_{c}^{2}
$$

is a pseudometric in $M_{c}-\{p\}$ with the same properties. 
We will need the following lemma, due to Robert Bryant.

Lemma [1, Proposition 4]. Let $d \tilde{s}^{2}$ be a pseudometric of constant Gaussian curvature $\widetilde{K}=1$ defined in a punctured disk $\Omega^{*}=\{\omega \in \mathbb{C}-\{0\} ;|\omega|<1\}$ of the complex plane $\mathbb{C}$. Assume that the area of $\Omega^{*}$ in the metric $d \tilde{s}^{2}$ is finite. Then there exist a number $\varepsilon>0$, an isothermal parametrization $z$ in $\Omega_{\varepsilon}=\{\omega \in \mathbb{C} ;|\omega|<\varepsilon\}$, with $z(0)=0$, and a real number $\beta>-1 / 2$ such that in $\Omega_{\varepsilon}-\{0\}$,

$$
d \tilde{s}^{2}=\frac{4(1+2 \beta)^{2}(z \bar{z})^{2 \beta}}{\left\{1+(z \bar{z})^{2 \beta+1}\right\}^{2}} d z d \bar{z}
$$

Furthermore, $\beta$ is unique and $z$ is unique up to a rotation.

Remark 1. The above lemma applies in the case of a complete minimal surface of $R^{3}$ with finite total curvature, as can be seen by considering $d \tilde{s}^{2}=-K d s^{2}$, where $d s^{2}$ is the induced metric. In this case, $\beta$ is an integer and $d \tilde{s}^{2}$ extends to a pseudometric in a neighborhood of the origin (cf. [1]).

It follows form the lemma that, in a neighborhood $U$ of $p$ in $M_{c}$, there exists an isothermal parameter $z$, with $z(p)=0$, and a number $\beta>-1 / 2$ such that in $U-\{p\}$

$$
F^{*} d \tilde{s}^{2}=-K \lambda d s_{c}^{2}=-K \lambda \nu d z d \bar{z},
$$

where, as a function of the parameter $z$,

$$
-K \lambda \nu=\frac{4(1+2 \beta)^{2}(z \bar{z})^{2 \beta}}{\left\{1+(z \bar{z})^{2 \beta+1}\right\}^{2}}
$$

We notice that $-K \lambda \nu$ is constant along the circles $z \bar{z}=$ const.

We have two cases to consider:

Case 1. $\beta \geq 0$. In this case, it follows from the above expression that $-K \lambda \nu \rightarrow$ 0 as $z \rightarrow 0$. Thus $-K \lambda$ is bounded in $M_{c}$. Set

$$
C_{0}=\sup _{M_{c}}(-2 K \lambda)
$$

and consider the operator $L_{0}=\Delta_{c}+C_{0}$. Since $M_{c}$ is compact, the index of $L_{0}$ in $M_{c}$ is finite. On the other hand, given $f: M_{c}-\{p\} \rightarrow R$ with compact support,

$$
I_{L_{c}}(f)=\int_{M_{c}}\left(\left|\nabla_{c} f\right|^{2}+2 K \lambda f^{2}\right) d A_{c} \geq \int_{M_{c}}\left(\left|\nabla_{c} f\right|^{2}-C_{0} f^{2}\right) d A_{c}=I_{L_{0}}(f) .
$$

Thus, if $f$ is such that $I_{L_{c}}(f)<0$, then $I_{L_{0}}(f)<0$. It follows that

$$
\text { Ind }_{M_{c}-\{p\}} L_{c} \leq \operatorname{Ind}_{M_{c}} L_{0}<\infty,
$$

and this proves the theorem in this case.

Remark 2. The above proof, together with Theorem $\mathrm{C}$, gives a proof of Theorem A that makes no use of the Gauss map (this corresponds to the case where 
$\beta$ is an integer; cf. Remark 1). The case $\beta \geq 0$ also includes various examples of surfaces with constant mean curvature 1 in $H^{3}(-1)$, to which the Gauss map does not extend (cf. [1, Example 1]).

Remark 3. The above proof also contains the following intrinsic theorem. Let $M^{2}$ be a surface with a complete Riemannian metric $d s^{2}$. Assume that the Gaussian curvature $K$ of $d s^{2}$ satisfies $K \leq 0$ and that $\int_{M}(-K) d A<\infty$. By Huber [5], $M$ is conformally equivalent to $M_{c}-\left\{p_{1}, \ldots, p_{k}\right\}$, where $M_{c}$ is a compact Riemann surface. Let $d s_{c}^{2}$ be a Riemannian metric in $M_{c}$ compatible with its conformal structure, and define $\lambda: M_{c}-\left\{p_{1}, \ldots, p_{k}\right\} \rightarrow R$ by $d s^{2}=$ $\lambda d s_{c}^{2}$. If $K \lambda$ is bounded in $M_{c}$, then the index of $L=\Delta-2 K$ in $M$ is finite. Furthermore, an upper bound for this index is the number (with multiplicities) of eigenvalues of $\Delta_{c}$ that are smaller than the supremum of $-2 K \lambda$ in $M_{c}$.

Case 2. $-1 / 2<\beta<0$. In this case, set

$$
0<1+2 \beta=\mu<1,
$$

and assume initially that $\mu=1 / n, n$ an integer,

Let $S^{2}$ be the unit sphere in $R^{3}$, and let $\zeta$ be an isothermal parameter in $S^{2}$ such that the canonical metric in $S^{2}$ is given by

$$
\frac{4}{(1+\zeta \bar{\zeta})^{2}} d \zeta d \bar{\zeta}
$$

Observe that the metric induced in $U-\{p\}$ by a branch of the map $h: U \rightarrow S^{2}$ given by

$$
\zeta=h(z)=z^{1 / n}
$$

with $1 / n=1+2 \beta$, is

$$
\frac{4}{\left(1+(z \bar{z})^{1 / n}\right)^{2}}\left|h^{\prime}(z)\right|^{2} d z d \bar{z}=\frac{4(1+2 \beta)^{2}(z \bar{z})^{2 \beta}}{\left\{1+(z \bar{z})^{1+2 \beta}\right\}^{2}} d z d \bar{z} .
$$

It follows that $h^{-1}(\zeta)=\zeta^{n}$ is a branched isometric covering of a domain of $S^{2}$ onto $U$ with the metric $-K \lambda d s_{c}^{2}$.

Now let $M_{0} \subset M_{c}-\{p\}$ be a domain with compact closure and regular boundary $\partial M_{0}$. We first choose $M_{0}$ such that $\partial M_{0}$ is a simple closed curve contained in $U$ and that $-2 K \lambda \nu=$ const. $=C_{0}>0$ along $\partial M_{0}$. By hypothesis, $-K \lambda \nu$ is not bounded, so we can choose $M_{0}$ sufficiently large so that $C_{0}>4 / n$. We will define an operator $G$ in $M_{c}$ by

$$
G= \begin{cases}\frac{1}{n} \Delta_{c}-2 K \lambda & \text { in } M_{0} \\ \frac{1}{2} \Delta_{c}+2 C_{0} / \nu & \text { in } M_{c}-M_{0} .\end{cases}
$$

Clearly, $\operatorname{Ind}_{M_{c}} G$ is finite. We want to show that

$$
\operatorname{Ind}_{M_{c}-\{p\}} L_{c} \leq \operatorname{Ind}_{M_{c}} G<\infty,
$$

and this will prove the theorem for $\beta<0$ and $\mu=1 / n$. 
For that purpose, let $f: M_{c}-\{p\} \rightarrow R$ be a function with compact support. We can assume that the support of $f$ contains $M_{0}$ and that the boundary of the support is a simple closed curve where $K \lambda \nu=$ const. Then

$$
\begin{aligned}
I_{H}(f) & =\int_{M_{0}}\left(\frac{1}{n}\left|\nabla_{c} f\right|^{2}+2 K \lambda f^{2}\right) d A_{c}+\int_{M_{c}-M_{0}}\left(\frac{1}{n}\left|\nabla_{c} f\right|^{2}-\frac{2 C_{0}}{\nu} f^{2}\right) d A_{c} \\
& \leq \int_{M_{0}}\left(\left|\nabla_{c} f\right|^{2}+2 K \lambda f^{2}\right) d A_{c}+\int_{M_{c}-M_{0}}\left(\frac{1}{n}\left|\nabla_{0} f\right|^{2}-2 C_{0} f^{2}\right) d z \wedge d \bar{z},
\end{aligned}
$$

where we have used $\nabla_{0}$ to denote the gradient of the flat metric.

Now let the plane $z$ be stereographically projected into the unit sphere $S^{2}$. Thus, the metric of $S^{2}$ is given by $\alpha d z d \bar{z}$, where $\alpha=4 /(1+z \bar{z})$. Thus the second term of the right-hand side of the above inequality can be estimated by

$$
\begin{aligned}
\int_{M_{c}-M_{0}}\left(\frac{1}{n}\left|\nabla_{0} f\right|^{2}-2 C_{0} f^{2}\right) d z d \bar{z} & =\int_{M_{c}-M_{0}}\left(\frac{1}{n}\left|\nabla_{S^{2}} f\right|^{2}-\frac{2 C_{0} f^{2}}{\alpha}\right) d A_{S^{2}} \\
& =n \int_{M_{c}-M_{0}}\left(\frac{1}{n}\left|\nabla_{c} f\right|^{2}+\frac{2 C_{0}}{\alpha} K \lambda f^{2}\right) d A_{c} \\
& \leq \int_{M_{c}-M_{0}}\left(\left|\nabla_{c} f\right|^{2}+\frac{2 C_{0} n}{4} K \lambda f^{2}\right) d A_{c} \\
& \leq \int_{M_{c}-M_{0}}\left(\left|\nabla_{c} f\right|^{2}+2 K \lambda f^{2}\right) d A_{c},
\end{aligned}
$$

where in the second equality we have used the fact that, locally, the metric of $S^{2}$ covers $n$ times the metric $-K \lambda d s_{c}^{2}$ of $M_{c}$, and in the two last inequalities we used the fact that $\alpha<4$ and $C_{0}>4 / n$.

It follows that

$$
\begin{aligned}
I_{G}(f) & \leq \int_{M_{0}}\left(\left|\nabla_{c} f\right|^{2}+2 K \lambda f^{2}\right) d A_{c}+\int_{M_{c}-M_{0}}\left(\left|\nabla_{c} f\right|^{2}+2 K \lambda f^{2}\right) d A_{c} \\
& =I_{L_{c}}(f),
\end{aligned}
$$

hence, $\operatorname{Ind}_{M_{c}-\{p\}} L_{c} \leq \operatorname{Ind}_{M_{c}} G$, as we wished.

Finally we consider the case in which $\mu \neq 1 / n$ for every integer $n$. Choose an integer $n_{0}$ such that $1 / n_{0}<\mu$, and define $\beta_{0}$ by

$$
1+2 \beta=\mu>1 / n_{0}=1+2 \beta_{0} .
$$

Then, in a neighborhood of $z=0$,

$$
q_{0}=\frac{4\left(1+2 \beta_{0}\right)^{2}(z \bar{z})^{2 \beta_{0}}}{\left\{1+(z \bar{z})^{1+2 \beta_{0}}\right\}^{2}}>\frac{4(1+2 \beta)^{2}(z \bar{z})^{2 \beta}}{\left[1+(z \bar{z})^{1+2 \beta}\right]^{2}}=q .
$$

Thus, if $f$ is a function as above and $U$ is sufficiently small,

$$
\int_{U}\left(\left|\nabla_{0} f\right|^{2}-2 q_{0} f^{2}\right) d z \wedge d \bar{z}<\int_{U}\left(\left|\nabla_{0} f\right|^{2}-2 q f^{2}\right) d z \wedge d \bar{z} .
$$


It follows that $\operatorname{Ind}_{M_{c}-\{p\}} L_{c}$ computed with $\mu<1 / n_{0}$ is smaller than or equal to $\operatorname{Ind}_{M_{c}} \tilde{G}$, where

$$
\widetilde{G}= \begin{cases}\frac{1}{n_{0}} \Delta_{c}-2 K \lambda & \text { in } M_{0} \\ \frac{1}{n_{0}} \Delta_{c}+2 C_{0} / \nu & \text { in } M_{c}-M_{0} .\end{cases}
$$

Since the latter is finite, the theorem is completely proved.

\section{REFERENCES}

1. R. Bryant, Surfaces of mean curvature one in hyperbolic spaces, Astérisque (to appear).

2. D. Fischer-Colbrie, On complete minimal surfaces with finite Morse index in three-manifolds, Invent. Math. 82 (1985), 121-132.

3. R. Gulliver, Index and total curvature of complete minimal surfaces, Proc. Sympos. Pure Math. 44 (1986), 207-211.

4. R. Gulliver and B. Lawson, The structure of stable minimal hypersurface near a singularity, Proc. Sympos. Pure Math. 44 (1986), 213-237.

5. A. Huber, On subharmonic functions and differential geometry in the large, Comment. Math. . Helv. 32 (1957), 13-72.

6. A. M. da Silveira, Stability of complete noncompact surfaces with constant mean curvature, Math. Ann. 277 (1987), 629-638.

Instituto de Matemática Pura e Aplicada, Estrada Dona Castorina, 110 Jardim BotÂNICO, Rio de JANEIRo, R.J. BRAZIL 